\title{
Conversion therapy for peritoneal lavage cytology-positive type 4 and large type 3 gastric cancer patients selected as candidates for R0 resection by diagnostic staging laparoscopy
}

\author{
Itaru Yasufuku ${ }^{1} \cdot$ Souya Nunobe ${ }^{1} \odot$. Satoshi Ida ${ }^{1} \cdot$ Koshi Kumagai $^{1} \cdot$ Manabu Ohashi $^{1} \cdot$ Naoki Hiki $^{1} \cdot$ Takeshi Sano $^{1}$
}

Received: 24 April 2019 / Accepted: 18 July 2019 / Published online: 26 July 2019

(c) The International Gastric Cancer Association and The Japanese Gastric Cancer Association 2019

\begin{abstract}
Background The long-term outcomes of type 4 and large type 3 gastric cancer patients with positive peritoneal lavage cytology (CY1) remain unsatisfying. We evaluated our treatment strategy of conversion therapy for CY1 patients without peritoneal dissemination (P0).

Methods Diagnostic staging laparoscopy (DSL) was performed before treatment. Chemotherapy was applied for DSL-diagnosed P0CY1. The re-evaluation of peritoneal metastasis by staging laparoscopy (re-SL) was performed when a response to chemotherapy was identified by gastroscopy and/or CT. Gastrectomy with radical lymphadenectomy was applied as conversion therapy when peritoneal lavage cytology-negative (CY0) and P0 were diagnosed with re-SL, with the aim of achieving R0 resection. Chemotherapy was continued as palliative treatment in patients for whom re-SL was not applicable or when re-SL did not confirm P0CY0. The long-term outcomes were retrospectively evaluated.

Results Between 2009 and 2015, 214 patients with type 4 and large type 3 gastric cancer underwent DSL in the Cancer Institute Hospital. Thirty-nine patients were initially diagnosed with P0CY1. Seven patients received palliative gastrectomy first due to outlet obstruction or other reasons. Thirty-two patients received chemotherapy first. Among them, 13 patients underwent gastrectomy as conversion therapy and 19 were treated with palliative chemotherapy. The 3-year survival rate of patients who underwent conversion therapy, palliative chemotherapy and palliative gastrectomy was $76.9 \%$ [95\% confidence interval (CI) $47.8-92.4 \%$ ], $10.5 \%$ (95\% CI 1.9-42.3\%), and 0\%, respectively.
\end{abstract}

Conclusion Conversion therapy might be a promising treatment for P0CY1 type 4 and large type 3 gastric cancer patients. Re-SL was useful for selecting candidates for R0 resection.

Keywords Type 4 gastric cancer · Large type 3 gastric cancer · Positive peritoneal lavage cytology · Conversion therapy · Diagnostic staging laparoscopy

\section{Introduction}

Type 4 gastric cancer has poorer prognosis than that of other gastric cancer types [1,2]. Large type 3 gastric cancer with a tumor diameter $\geq 8 \mathrm{~cm}$ has oncological characteristics that are similar to those of type 4 gastric cancer [3]. Such tumors show a high frequency of peritoneal metastasis [including peritoneal dissemination (P1) and positive peritoneal

Souya Nunobe

souya.nunobe@jfcr.or.jp

1 Department of Gastroenterological Surgery, Cancer Institute Hospital, Japanese Foundation for Cancer Research, 3-8-31 Ariake, Koto-ku, Tokyo 135-8551, Japan cytology (CY1)] at initial diagnosis [4]. CY1, which is one of the factors of distant metastasis (M1) and which is classified into stage IV in the UICC, AJCC and the Japanese classification of gastric carcinoma [5-7], has been reported to be a prognostic factor for gastric cancer [8].

The standard treatment for gastric cancer patients with M1 is systemic chemotherapy [9, 10]. Recently, several studies have reported that surgery combined with perioperative chemotherapy, which was mainly divided into adjuvant chemotherapy and neoadjuvant chemotherapy (NAC), may have a survival benefit in gastric cancer patients with CY1 [11-13]. Another treatment strategy for advanced gastric cancer is conversion therapy, which is defined as a surgical treatment aimed at achieving R0 resection after chemotherapy for tumors that were originally unresectable 
or marginally resectable for technical and/or oncological reasons $[14,15]$.

Diagnostic staging laparoscopy (DSL) is a safe and useful procedure for diagnosing peritoneal metastasis in gastric cancer patients [16]. Since peritoneal metastasis is difficult to diagnose based on radiological examinations alone, DSL should be considered at the time of initial staging before deciding on the treatment strategy for type 4 and large type 3 gastric cancer [4]. Moreover, DSL is useful for evaluating the intracorporeal response to chemotherapy in gastric cancer patients.

In the current study, we evaluated the survival benefit of our treatment strategy of conversion therapy, utilizing DSL for the assessment of peritoneal metastasis, in type 4 and large type 3 gastric cancer initially diagnosed as CY1 without peritoneal dissemination $(\mathrm{P} 0)$ or other distant metastasis.

\section{Patients and methods}

\section{Patients}

This retrospective study included 214 consecutive patients who were diagnosed with type 4 and large type 3 gastric cancer at the Cancer Institute Hospital between June 2009 and December 2015. Macroscopic type 4 or type 3 disease was diagnosed using gastroscopy (GS) and based on the Japanese classification of gastric carcinoma 14th edition and 3rd English edition [7]. In detail, type 4 cancer was characterized by the presence of tumors without marked ulcerations or raised margins, gastric wall thickening and induration, and unclear margins. Type 3 cancer was characterized by the presence of ulcerated tumors with raised margins, surrounded by a thickened gastric wall without clear margins [7]. Tumor size was also estimated with GS.

\section{The treatment strategy for type 4 and large type 3 gastric cancer in our institution}

In our institution, the treatment strategy for type 4 and large type 3 gastric cancer was as follows: DSL was performed first when preoperative imaging examinations did not detect any factors associated with unresectable disease (e.g., definitive peritoneal metastasis, multiple liver metastases, lung metastasis, or a large amount of ascites). A few paraaortic lymph node metastases were considered to be resectable [17]. Gastrectomy with D2 lymphadenectomy was performed for DSL-diagnosed P0 and peritoneal lavage cytology-negative (CY0) disease. Palliative chemotherapy was applied in cases in which $\mathrm{P} 1$ was diagnosed by DSL. Chemotherapy was applied with the aim of achieving conversion therapy for DSL-diagnosed P0CY1. GS and/or CT were performed after every 2 or 3 chemotherapy cycles to evaluate the response of chemotherapy. When response to first-line chemotherapy was observed, re-evaluation of peritoneal metastasis with diagnostic staging laparoscopy (re-SL) was performed. The response to chemotherapy was evaluated using GS and/or CT. Primary tumor shrinkage, improvement in gastric wall extensibility with air inflation on GS, or metastatic lymph node shrinkage on CT were evaluated by comparison with pretreatment observations. Response to chemotherapy was confirmed when either one or more of the above-mentioned criteria were fulfilled and re-SL was applied. In case showing lymph node enlargement despite primary tumor shrinkage or the reverse, re-SL was not applied and disease progression was considered present. When the re-SL-diagnosed peritoneal cytology changed to negative and no new metastatic lesions were identified, gastrectomy with radical lymphadenectomy was performed in a couple of weeks as conversion therapy with the aim of achieving R0 resection. When re-SL showed that CY1 remained, chemotherapy was continued. When P1 was diagnosed, second-line chemotherapy was initiated due to tumor progression. Before July 2010, exploratory laparotomy was performed instead of re-SL just before gastrectomy; however, it was replaced with re-SL as futile laparotomy in which P0CY0 was not obtained was considered to be invasive in patients in whom chemotherapy should have been continued.

\section{Treatment}

The details of the first-line chemotherapy regimens for patients with CY1 were as follows: HER2-negative disease: (1) S-1 plus cisplatin (SP): S-1 [the dose was $80 \mathrm{mg}$ / day for body surface area (BSA) $<1.25 \mathrm{~m}^{2}, 100 \mathrm{mg}$ /day for BSA $\geq 1.25$ to $<1.5 \mathrm{~m}^{2}$ and $120 \mathrm{mg} /$ day for BSA $\geq 1.5 \mathrm{~m}^{2}$ ] was administered orally, twice daily for 3 consecutive weeks followed by a 1- or 2-week rest, and cisplatin $\left(60 \mathrm{mg} / \mathrm{m}^{2}\right)$ was administered intravenously on day 8. (2) S-1 plus oxaliplatin (SOX): S-1 was administered orally, twice daily for 2 consecutive weeks followed by a 1-week rest, and oxaliplatin $\left(100 \mathrm{mg} / \mathrm{m}^{2}\right)$ was administered intravenously on day 1. HER2-positive disease: (1) capecitabine, cisplatin and trastuzumab $(\mathrm{XP}+\mathrm{T})$ : capecitabine $1000 \mathrm{mg} / \mathrm{m}^{2}$ was administered orally twice a day for 14 days followed by a 1 -week rest. Cisplatin $\left(80 \mathrm{mg} / \mathrm{m}^{2}\right)$ was administered on day 1 by intravenous infusion. Trastuzumab was administered by intravenous infusion at a dose of $8 \mathrm{mg} / \mathrm{kg}$ on day 1 of the first cycle, followed by $6 \mathrm{mg} / \mathrm{kg}$ every 3 weeks. (2) S-1, oxaliplatin and trastuzumab (SOX + T): S-1 [the dose was $80 \mathrm{mg} /$ day for body surface area (BSA) $<1.25 \mathrm{~m}^{2}, 100 \mathrm{mg} /$ day for $\mathrm{BSA} \geq 1.25$ to $<1.5 \mathrm{~m}^{2}$ and $120 \mathrm{mg} /$ day for BSA $\geq 1.5 \mathrm{~m}^{2}$ ] was administered orally, twice daily for 2 consecutive weeks, and oxaliplatin at $100 \mathrm{mg} / \mathrm{m}^{2}$ was administered by intravenous infusion. Trastuzumab was administered by intravenous 
infusion at a dose of $8 \mathrm{mg} / \mathrm{kg}$ on day 1 of the first cycle, followed by $6 \mathrm{mg} / \mathrm{kg}$ every 3 weeks.

Gastrectomy with D2 or D2 + lymphadenectomy [9] was performed as conversion therapy when a clinical response was seen after chemotherapy and re-SL diagnosed P0CY0. Splenectomy was performed for patients in whom lymph node metastasis was suspected to the splenic hilum area or in patients whose tumor massively invaded the greater curvature of the upper gastric body or fornix. D2 + paraaortic lymphadenectomy was performed when paraaortic lymph node metastasis was suspected before chemotherapy.

Postoperative chemotherapy was proposed after gastrectomy. S-1 was administered for 1 year after R0 resection. S-1 was administered until progression was identified in patients whose surgery resulted in $\mathrm{R} 1$ resection. When recurrence after R0 resection or progression after R1 resection was identified, palliative chemotherapy was performed.

\section{Assessment and statistical methods}

The clinical and pathological diagnosis of tumor depth, lymph node metastasis and distant metastasis and the histological response of the primary tumor were classified according to the Japanese classification of gastric carcinoma 14th edition and 3rd English edition [7]. Histological responses were classified into 4 grades: grade 0 , no evidence of effect; grade 1a, viable tumor cell occupying two-thirds or more of the tumorous area; grade $1 \mathrm{~b}$, viable tumor cells occupying from one-third to less than two-thirds of the tumorous area; grade 2, viable tumor cells occupying less than one-third of the tumorous area; and grade 3 , no viable tumor cells.

The baseline characteristics of patients who underwent conversion therapy and those who could not undergo conversion therapy were compared by Fisher's exact test or the Wilcoxon signed-rank test, as appropriate. The overall survival rates were estimated by the Kaplan-Meier method. The overall survival rates of groups divided according to treatment (conversion therapy, palliative chemotherapy, and resection first) were compared with the log-rank test. $P$ values of $<0.05$ were considered to indicate statistical significance. All statistical analyses were performed using the JMP software program (SAS Institute Inc., Cary, NC, USA).

\section{Results}

\section{Patient characteristics}

A flowchart of the assessment and the number of patients who received each treatment is shown in Fig. 1. Two hundred fourteen type 4 and large type 3 gastric cancer patients underwent DSL. Among the 214 patients, 39 patients were initially diagnosed with P0CY1. The characteristics of the 39 patients are shown in Table 1. Among these 39 patients, 7 patients underwent palliative gastrectomy first (surgery-first group) because 3 patients presented gastric outlet obstruction, 3 patients declined chemotherapy first and 1 patient was enrolled in another randomized-controlled clinical trial of neoadjuvant chemotherapy and was allocated to the group in which gastrectomy was applied first without neoadjuvant chemotherapy. Chemotherapy was administered first, aiming at conversion therapy in the other 32 patients. Among these patients, 18 patients showed a response to chemotherapy in GS or CT examinations and underwent re-SL. P0CY0 was confirmed in 13 of these patients by re-SL [conversion rate was $40.6 \%$ (13/32)]. Thirteen patients underwent gastrectomy with radical lymphadenectomy as conversion therapy (conversion therapy group). On the other hand, 14 of 32 patients who did not show a certain response to chemotherapy and 5 patients who did not obtain P0CY0 though re-SL underwent further chemotherapy as a palliative treatment (palliative chemotherapy group).

Comparison of characteristics between the patients who underwent conversion therapy and palliative chemotherapy is shown in Table 2 . There were no statistically significant differences in the age, sex, macroscopic type, histological type, preoperative $\mathrm{T}$ and $\mathrm{N}$ grade, or preoperative tumor markers between the two groups.

The first-line chemotherapy regimens administered after the initial diagnosis of P0CY1 are shown in Table 3. Doublet agent chemotherapies consisting of fluoropyrimidine agents and platinum agents were administered to all patients in the conversion therapy group. Regarding the preoperative chemotherapy treatment period, two or three cycles of SP regimen, three cycles of SOX regimen, three cycles of $\mathrm{SOX}+\mathrm{T}$ regimen and three cycles of $\mathrm{XP}+\mathrm{T}$ regimen were administered. In contrast, $\mathrm{S}-1$ monotherapy was administered to 2 patients (aged 89 and 83 years at diagnosis) due to their advanced age and 5-FU plus leucovorin (FL) therapy was administered to 1 patient due to gastric outlet obstruction caused by the tumor and the fact that oral agents could not be administered (the FOLFOX regimen was not used in practice in Japan at that time). Another 3 patients received chemotherapy in another hospital.

\section{Surgical outcomes}

The surgical outcomes of conversion therapy are shown in Table 4. Twelve out of 13 patients were treated with total gastrectomy. R0 resection was achieved in 10 patients. Among 3 patients for whom R0 resection could not be achieved, the proximal margin was positive for cancer due to esophageal invasion in one patient, the distal margin was positive for cancer due to duodenal invasion in one patient, and peritoneal cytology turned to positive when gastrectomy 
Fig. 1 Flowchart of patient recruitment. P0, Absence of peritoneal dissemination; $\mathrm{P} 1$, presence of peritoneal dissemination; $\mathrm{CY} 0$, negative peritoneal cytology; CY1, positive peritoneal cytology

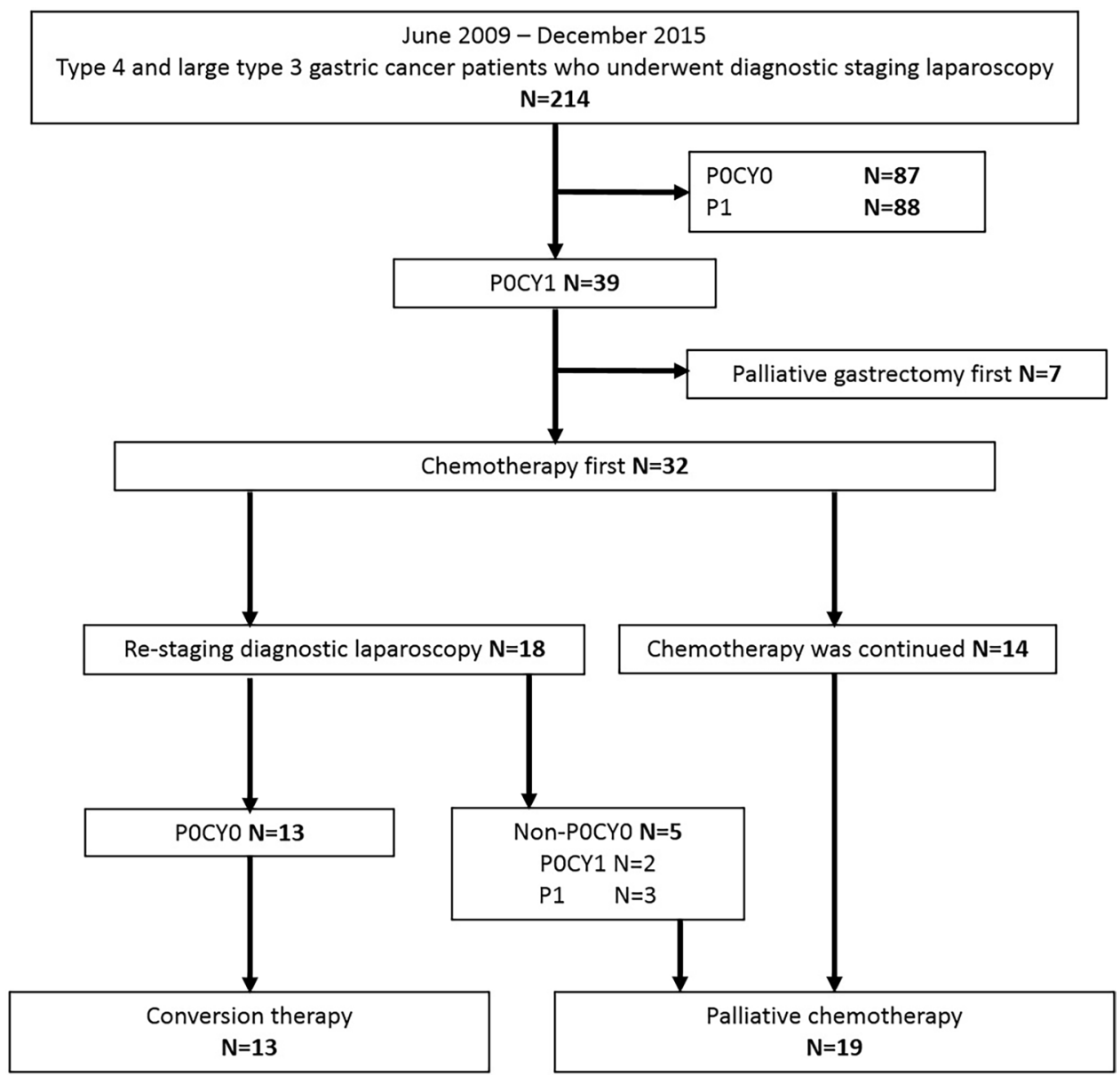

was performed, although re-SL had confirmed CYO in one patient.

Regarding postoperative complications, 5 patients experienced Clavien-Dindo grade III complications, including leakage of a duodenal stump treated with a conservative procedure. There were no treatment-related deaths.

A histopathological examination of the resected primary tumor showed that the response to chemotherapy was Grade 1a in 3 patients, Grade $1 \mathrm{~b}$ in 5 patients, and Grade 2 in 5 patients. No patients showed a Grade 3 response.

\section{The survival}

The median follow-up length was 46.5 months (range 10.5-108.5) in the conversion therapy group, 16.3 months (range 5.1-47.1) in the palliative chemotherapy group and 18.0 months (range 4.8-36.8) in the surgery-first group.

Recurrence or progression after conversion therapy is shown in Table 5. Six patients had recurrence after R0 resection. All patients whose surgery resulted in $\mathrm{R} 1$ resection had tumor progression.

The median survival time (MST) of all 39 patients initially diagnosed with P0CY1 and treated with our strategy was 24.1 months [95\% confidence interval (CI) 16.3-37.9 months] from the diagnosis (Fig. 2a). The 3 - and 5-year survival rates of all patients were $35.5 \%$ (95\% CI 22.1-51.6\%) and 28.9\% (95\% CI 16.5-45.6\%), respectively. The overall survival in each group is shown in Fig. 2b. The MST was 108.5 months in the conversion therapy group (95\% CI 29.6-108.5 months), including 5 patients who survived for more than 5 years, 16.3 months (95\% CI 8.8-26.2 months) in the palliative chemotherapy group and 18.0 months (95\% CI 4.8-31.1 months) in the surgery-first group $(P=0.0005)$. The 3 -year survival rates in the conversion therapy, palliative chemotherapy and surgery-first groups were 76.9\% (95\% CI 47.8-92.4 months), $10.5 \%$ (95\% CI $1.9-42.3 \%$ ) and $0 \%$, respectively. The progression-free survival (PFS) in each group is shown in Fig. 2c. The median PFS was 27.9 months (95\% CI 11.4 months to not reached) in the conversion therapy group, 8.1 months (95\% CI 4.1-14.1 months) in the palliative chemotherapy group and 14.9 months $(95 \% \mathrm{CI}$ $3.2-16.3$ months $)$ in the surgery-first group $(P=0.0017)$, respectively. 
Table 1 Background characteristics of 39 patients initially diagnosed with P0CY1 disease

\begin{tabular}{|c|c|}
\hline Characteristics & $N=39$ \\
\hline Age $\left(\right.$ years) ${ }^{\mathrm{a}}$ & $66(21-89)$ \\
\hline \multicolumn{2}{|l|}{ Sex } \\
\hline Male & $16(41 \%)$ \\
\hline Female & $23(59 \%)$ \\
\hline \multicolumn{2}{|l|}{ Macroscopic type } \\
\hline Type 3 & $7(18 \%)$ \\
\hline Type 4 & $32(82 \%)$ \\
\hline \multicolumn{2}{|l|}{ Main location } \\
\hline $\mathrm{U}$ & $2(5 \%)$ \\
\hline M & $7(18 \%)$ \\
\hline $\mathrm{L}$ & $1(3 \%)$ \\
\hline Whole stomach & $29(74 \%)$ \\
\hline \multicolumn{2}{|l|}{ Histological type } \\
\hline Differentiated type & 0 \\
\hline Mixed type & $18(46 \%)$ \\
\hline Undifferentiated type & $21(54 \%)$ \\
\hline \multicolumn{2}{|l|}{ Clinical $\mathrm{T}$ grade } \\
\hline $\mathrm{T} 3$ & 0 \\
\hline $\mathrm{T} 4 \mathrm{a}$ & $38(97 \%)$ \\
\hline $\mathrm{T} 4 \mathrm{~b}$ & $1(3 \%)$ \\
\hline \multicolumn{2}{|l|}{ Clinical N grade } \\
\hline No & $12(31 \%)$ \\
\hline N1 & $15(38 \%)$ \\
\hline $\mathrm{N} 2$ & $7(18 \%)$ \\
\hline N3 & $5(13 \%)$ \\
\hline \multicolumn{2}{|l|}{ Tumor marker $^{\mathrm{a}}$} \\
\hline $\mathrm{CEA}^{\mathrm{a}}$ & $3.3(0.5-134.3)$ \\
\hline CA19-9a & $9.8(2.0-50,000)$ \\
\hline $\mathrm{CA} 125^{\mathrm{a}}$ & $14.5(3.0-76.8)$ \\
\hline
\end{tabular}

CEA carcinoembryonic antigen

${ }^{a}$ Values are expressed as the median (range)

\section{Discussion}

Among 32 P0CY1 type 4 and large type 3 gastric cancer patients who underwent intensive chemotherapy, 18 patients presented a clinical response and underwent re-SL. POCY0 was confirmed in 13 patients and 5 patients were judged to be unsuitable for surgical resection by re-SL. Conversion therapy was applied to 13 patients and the 3 -year survival rate was $76.9 \%$, which was a promising result.

No standard treatment has been established for CY1 gastric cancer patients without any other non-curative factors. Several studies have reported that surgery combined with perioperative chemotherapy may provide a survival benefit to gastric cancer patients with P0CY1 [11-13]. Kodera et al. $[12,18]$ demonstrated that gastrectomy with lymph node dissection followed by adjuvant chemotherapy with
S-1 provided a 5-year overall survival rate of $26 \%$ and a relapse-free survival rate of $21 \%$ in P0CY1 patients. In the study, however, $38 \%$ of the patients had differentiated type gastric cancer, and 57\% patients were treated with distal gastrectomy. In other words, the background of the patients in the study differed from that of the patients with type 4 and large type 3 gastric cancer. Aizawa et al. [13] demonstrated that gastrectomy after induction chemotherapy for patients initially diagnosed with CY1 without any other non-curative factors provided a 5-year overall survival rate of $25 \%$ and reported that better survival could be expected when R0 resection could be achieved. However, approximately $50 \%$ of surgeries resulted in non-curative resection, and the prognosis of these cases was dismal. In the current study, although all patients had type 4 or large type 3 gastric cancer and gastrectomy was only performed for patients whose peritoneal lavage cytology turned negative, with the aim of achieving $\mathrm{R} 0$ resection and ineffective resection was avoided, the MST of all patients was 24.1 months, which was acceptable result. Furthermore, a long survival time could be expected in patients who underwent conversion therapy.

The evaluation of peritoneal metastasis before conversion to surgical resection is important. Aizawa et al. reported that among patients who were initially diagnosed with CY1, without any other distant metastasis, peritoneal lavage cytology was converted to negative after chemotherapy in $48.9 \%$ of patients. In contrast, new peritoneal dissemination was reported to appear after chemotherapy in $19.2 \%$ of the patients [13]. Reevaluation before surgical resection would avoid half of the surgeries that would result in R1 or R2 resection.

We consider that DSL is a preferable procedure for evaluating peritoneal metastasis in gastric cancer patients. DSL was reported to be a common, less invasive, safe and useful procedure for diagnosing peritoneal metastasis of gastric cancer [16]. Irino et al. [19] reported that the accuracy of DSL was $92 \%$ and DSL is highly influential in clinical decision-making for gastric cancer treatment. On the other hand, DSL had a non-negligible problem with false-negative results in the diagnosis of peritoneal metastasis. The falsenegative rate was reported to be $10.6-17.2 \%$ in previous studies $[4,19]$. In the current study, one patient was diagnosed with CY1 when conversion surgery was performed after the confirmation of CYO by re-SL. The influence of preoperative chemotherapy on the diagnosis of peritoneal metastasis by DSL was unclear. In patients initially diagnosed with CY1, cancer cells would be decreased in the abdominal cavity by preoperative chemotherapy, which would make an accurate diagnosis of washing cytology more difficult.

Selecting patients who should proceed to re-SL during first-line chemotherapy is also important. Blank et al. [20] reported that preoperative evaluation of the response to 
Table 2 Background characteristics of patients who underwent conversion therapy and those who underwent palliative chemotherapy

\begin{tabular}{|c|c|c|c|}
\hline Characteristics & Conversion therapy, $N=13$ & $\begin{array}{l}\text { Palliative chemotherapy, } \\
N=19\end{array}$ & $P$ value \\
\hline Age (years) ${ }^{\mathrm{a}}$ & $60(21-75)$ & $66(40-89)$ & 0.19 \\
\hline Sex & & & 0.47 \\
\hline Male & $4(31 \%)$ & $9(47 \%)$ & \\
\hline Female & $9(69 \%)$ & $10(53 \%)$ & \\
\hline Macroscopic type & & & 0.37 \\
\hline Type 3 & $3(23 \%)$ & $2(11 \%)$ & \\
\hline Type 4 & $10(77 \%)$ & $17(89 \%)$ & \\
\hline Histological type & & & 0.27 \\
\hline Differentiated type & 0 & 0 & \\
\hline Mixed type & $3(23 \%)$ & $9(47 \%)$ & \\
\hline Undifferentiated type & $10(77 \%)$ & $10(53 \%)$ & \\
\hline \multicolumn{4}{|l|}{ Clinical T grade } \\
\hline $\mathrm{T} 3$ & 0 & 0 & \\
\hline $\mathrm{T} 4 \mathrm{a}$ & $13(100 \%)$ & $19(100 \%)$ & \\
\hline $\mathrm{T} 4 \mathrm{~b}$ & 0 & 0 & \\
\hline Clinical N grade & & & 0.28 \\
\hline No & $6(46 \%)$ & $5(26 \%)$ & \\
\hline $\mathrm{N}+$ & $7(54 \%)$ & $14(74 \%)$ & \\
\hline N1 & 4 & 8 & \\
\hline $\mathrm{N} 2$ & 1 & 4 & \\
\hline N3 & 2 & 2 & \\
\hline \multicolumn{4}{|l|}{ Tumor marker } \\
\hline $\mathrm{CEA}^{\mathrm{a}}$ & $1.7(0.5-22.2)$ & $3.7(0.5-134.3)$ & 0.22 \\
\hline CA19-9 $9^{a}$ & $7.0(2.0-415.8)$ & $11.3(2.0-50,000)$ & 0.14 \\
\hline $\mathrm{CA} 125^{\mathrm{a}}$ & $16.2(5.6-62.8)$ & $17.9(3.1-76.8)$ & 0.86 \\
\hline
\end{tabular}

CEA carcinoembryonic antigen

${ }^{a}$ Values are expressed as the median (range)
Table 3 First-line chemotherapy regimens administered to P0CY1 type 4 and large type 3 gastric cancer patients according to treatment

\begin{tabular}{lll}
\hline & Conversion therapy, $N=13$ & $\begin{array}{l}\text { Palliative } \\
\text { chemotherapy, } \\
N=19\end{array}$ \\
\hline SP & 9 & 8 \\
SOX & 2 & 4 \\
SOX + T & 1 & 0 \\
XP+T & 1 & 1 \\
S-1 & 0 & 2 \\
FL & 0 & 1 \\
Unknown & 0 & 3 \\
\hline
\end{tabular}

$S P$ S-1 plus cisplatin, $S O X$ S-1 plus oxaliplatin, $S O X+T$ S-1 oxaliplatin and trastuzumab, $X P+T$ capecitabine, cisplatin and trastuzumab, $F L$ 5-FU plus leucovorin

chemotherapy by a combination of endoscopy and CT after chemotherapy was feasible in esophagogastric junctional cancer and gastric cancer patients treated with preoperative chemotherapy followed by surgical resection. They also reported that the clinical response was strongly correlated with the R0 resection rate, histological response and survival. In addition, it is unclear how the clinical response in patients with P0CY1 disease is to be evaluated. As there were few patients in whom the target lesion could be set in the case of P0CY1 disease, application of the Response Evaluation Criteria in Solid Tumors (RECIST) [21] for the evaluation of chemotherapy response was insufficient. In the current study, we evaluated the clinical response to chemotherapy with primary tumor or metastatic lymph node shrinkage compared to that recorded in the pretreatment examination, and we only performed re-SL during first-line chemotherapy in patients whose tumor showed a response. As a result, 18 patients showed a clinical response and the response rate was $56.3 \%$ (18/32). We applied re-SL for all 18 patients and $\mathrm{POCY} 0$ disease was confirmed in 13 (72.2\%) patients. This proportion $(72.2 \%)$ was relatively high. Nakamura et al. [22] reported that second-look staging laparoscopy confirmed P0CY0 disease presence in $66.7 \%$ of patients who were initially diagnosed with P0CY1 disease and underwent chemotherapy. In this report, second-look 
Table 4 Surgical procedures, pathological diagnosis and adjuvant chemotherapy regimens of 13 patients with conversion therapy

\begin{tabular}{|c|c|}
\hline & $N=13$ \\
\hline \multicolumn{2}{|l|}{ Operating procedure } \\
\hline Total gastrectomy & $12(92 \%)$ \\
\hline Distal gastrectomy & $1(8 \%)$ \\
\hline \multicolumn{2}{|l|}{ Combined resection } \\
\hline Spleen & $7(54 \%)$ \\
\hline Colon & $1(8 \%)$ \\
\hline Operation time $(\min )^{\mathrm{a}}$ & $278(179-620)$ \\
\hline Blood loss $(\mathrm{g})^{\mathrm{a}}$ & $450(160-3100)$ \\
\hline \multicolumn{2}{|l|}{ Lymph node dissection } \\
\hline D2 & $10(77 \%)$ \\
\hline $\mathrm{D} 2+$ & $3(23 \%)$ \\
\hline \multicolumn{2}{|c|}{ Postoperative complication (Clavien-Dindo grade) } \\
\hline Grade II & $2(15 \%)$ \\
\hline Intraabdominal infection & 1 \\
\hline Hepatic abscess & 1 \\
\hline Grade III & $5(38 \%)$ \\
\hline Intraabdominal infection & 3 \\
\hline Pneumothorax & 1 \\
\hline Leakage of duodenal stump & 1 \\
\hline \multicolumn{2}{|l|}{ Residual tumor } \\
\hline R0 & $10(77 \%)$ \\
\hline $\mathrm{R} 1$ & $3(23 \%)$ \\
\hline \multicolumn{2}{|l|}{ Pathological T grade } \\
\hline $\mathrm{T} 1$ & $2(15 \%)$ \\
\hline $\mathrm{T} 2$ & $1(8 \%)$ \\
\hline $\mathrm{T} 3$ & $2(15 \%)$ \\
\hline T4a & $7(54 \%)$ \\
\hline $\mathrm{T} 4 \mathrm{~b}$ & $1(8 \%)$ \\
\hline \multicolumn{2}{|l|}{ Pathological N grade } \\
\hline No & $3(23 \%)$ \\
\hline N1 & 0 \\
\hline $\mathrm{N} 2$ & $2(15 \%)$ \\
\hline N3 & $8(62 \%)$ \\
\hline \multicolumn{2}{|l|}{ Histological response } \\
\hline Grade 1a & $3(23 \%)$ \\
\hline Grade $1 \mathrm{~b}$ & $5(38 \%)$ \\
\hline Grade 2 & $5(38 \%)$ \\
\hline Grade 3 & 0 \\
\hline \multicolumn{2}{|l|}{ Adjuvant chemotherapy } \\
\hline S1 & $11(85 \%)$ \\
\hline $\mathrm{SOX}+$ trasutuzumab & $1(8 \%)$ \\
\hline None & $1(8 \%)$ \\
\hline
\end{tabular}

SOX S-1 plus oxaliplatin

${ }^{a}$ Values are expressed as the median (range)

staging laparoscopy was applied in patients without tumor progression; second-look laparoscopy was also applied to patients with tumor stability during chemotherapy.
Table 5 Numbers of patients with recurrence after conversion therapy and site of recurrence in patients who underwent $\mathrm{R} 0$ resection and $\mathrm{R} 1$ resection after conversion therapy

\begin{tabular}{lll}
\hline & $\begin{array}{l}\text { R0 resection, } \\
N=10\end{array}$ & R1 resection, $N=3$ \\
\hline Recurrence & $6(60 \%)$ & $3(100 \%)$ \\
Peritoneal dissemination & 2 & 2 \\
Ovary & 2 & 0 \\
Lymph node & 1 & 0 \\
Liver & 1 & 0 \\
Local recurrence & 0 & 1 \\
\hline
\end{tabular}

Considering these result, the application of re-SL only in patients whose tumor showed clinical response to chemotherapy may enable the confirmation of patients with POCY0 disease surely and efficiently.

The selection of patients as candidates for conversion therapy based on the clinical response to chemotherapy may also yield remarkable survival outcomes in patients with conversion therapy. Although the patients recruited to the study all had type 4 or large type 3 gastric cancer and more than $60 \%$ of the patients with conversion therapy were pathologically diagnosed with $\mathrm{N} 3$, the 3 -year survival rate of $76.9 \%$, as observed in the patients who underwent conversion therapy, was surprising. As mentioned above, clinical response to chemotherapy is associated with survival in gastric cancer [20]. The patients who underwent conversion therapy were those whose tumor showed response to chemotherapy. These tumor may also have shown better response to adjuvant chemotherapy after surgery or palliative chemotherapy on tumor relapse.

The optimal period of chemotherapy to obtain negative peritoneal lavage cytology in patients initially diagnosed with P0CY1 has not been established. Aizawa et al. [13] reported that $1-5$ cycles ( 1 cycle was mainly 3 or 4 weeks) of preoperative chemotherapy turned cytology negative in $48.9 \%$ patients (23/47). In the current study, 2 or 3 cycles of preoperative chemotherapy ( 1 cycle was 3 or 4 weeks) were administered and peritoneal lavage cytology turned from positive to negative in $40.6 \%$ of patients (13/32). Among patients for whom re-SL was not applicable or whose peritoneal lavage cytology did not turn negative, although re-SL was performed, no patients showed a further response to chemotherapy. Thus, the timing of the re-evaluation and consideration of conversion to surgical resection would be acceptable.

The present study was associated with several limitations. First, this was a retrospective, single institutional cohort study, with a relatively small patient population. Second, the observation period was relatively short. A considerable number of patients who received conversion therapy had recurrence after surgery; thus, mortality due to 

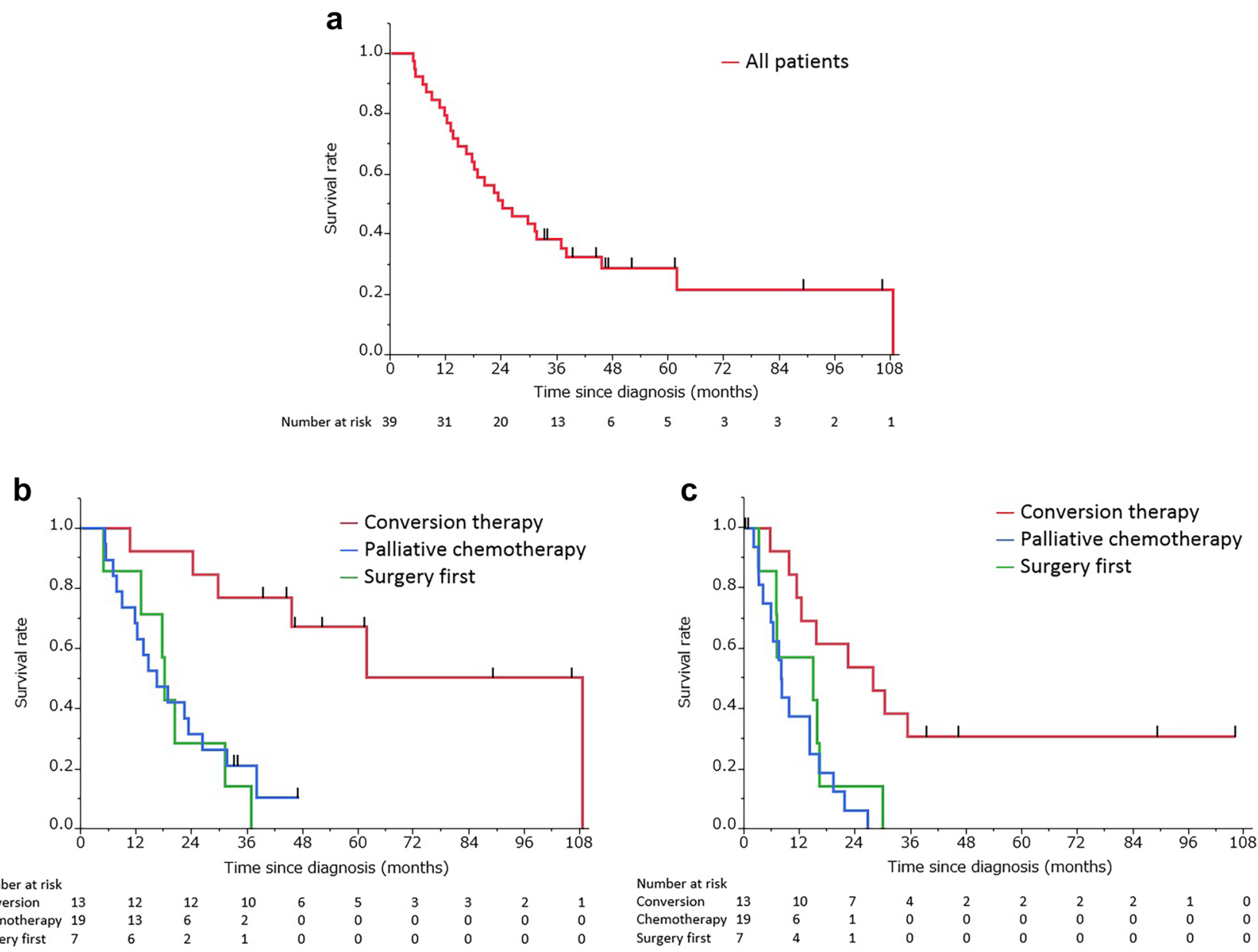

Fig. 2 a The Kaplan-Meier curve for overall survival of all patients treated with our strategy. b The Kaplan-Meier curve for overall survival of patients who underwent conversion therapy, palliative chemotherapy and surgery first (log-rank test, $P=0.0005)$. c The Kaplan-

Meier curve for progression-free survival of patients who underwent conversion therapy, palliative chemotherapy and surgery first (logrank test, $P=0.0017$ )

tumor recurrence would be increased. However, the observation period was more than three years in the conversion therapy group, and some patients survived for more than five years without recurrence. Even in patients with recurrence after surgery, relatively longer survival may be expected. Third, the chemotherapy regimens were not been unified. Although the optimal chemotherapeutic regimen has not been established for P0CY1 patients, combinations of a platinum agent and fluoropyrimidine agent were mainly applied in this study, and negative conversion of peritoneal lavage cytology was confirmed in approximately $40 \%$ patients (13/32). This result is considered acceptable. However, the palliative chemotherapy group included patients treated with S-1 monotherapy or the FL regimen because of their advanced age or the presence of gastric stenosis caused by the tumor. The difference in choice of first-line chemotherapy regimen was partly associated with the patient vulnerability or advanced tumor

status which may not have been confirmed statistically due to the small patient number. These biases would probably affect the tumor-response to chemotherapy, negative conversion of CY1 disease, and eventually the overall survival of the patients.

In conclusion, the long-term outcomes of P0CY1 type 4 and large type 3 gastric cancer patients whose peritoneal lavage cytology turned negative due to chemotherapy and who subsequently received conversion therapy may be promising. Applying re-SL to patients who showed clinical response to systemic chemotherapy may also be useful for selecting potential candidates for radical gastrectomy.

\section{Compliance with ethical standards}

Conflict of interest The authors declare that they have no conflict of interest. 
Human rights statement and informed consent All procedures followed were in accordance with the ethical standards of the responsible committee on human experimentation (institutional and national) and with the Helsinki Declaration of 1964 and later versions. As this study was a retrospective study and did not include any potentially identifiable patient data, informed consent to be included in the study was not obtained from the enrolled patients. The institutional review board gave the ethics approval for this retrospective study.

\section{References}

1. An JY, Kang TH, Choi MG, Noh JH, Sohn TS, Kim S. Borrmann type IV: an independent prognostic factor for survival in gastric cancer. J Gastrointest Surg. 2008;12:1364-9.

2. Li C, Oh SJ, Kim S, Hyung WJ, Yan M, Zhu ZG, et al. Macroscopic Borrmann type as a simple prognostic indicator in patients with advanced gastric cancer. Oncology. 2009;77:197-204.

3. Sasako M, Maruyama K, Kinoshita T, et al. E. Neoadjuvant chemotherapy for gastric cancer: indication and trial setting (in Japanese). Shokakigeka. 1992;15:159-67.

4. Miki Y, Tokunaga M, Tanizawa Y, Bando E, Kawamura T, Terashima M. Staging laparoscopy for patients with cM0, type 4, and large type 3 gastric cancer. World J Surg. 2015;39:2742-7.

5. Edge SBBD, Compton CC, Fritz AG, Greene FL, Trotti A. AJCC cancer staging manual. 7th ed. Berlin: Springer; 2009.

6. Sobin LGM, Wittekind C. TNM classification of malignant tumours. 7th ed. Wiley-Blackwell: Hoboken; 2009.

7. Japanese classification of gastric carcinoma: 3rd English edition. Gastr Cancer. 2011;14:101-12.

8. Miyashiro I, Takachi K, Doki Y, Ishikawa O, Ohigashi H, Murata $\mathrm{K}$, et al. When is curative gastrectomy justified for gastric cancer with positive peritoneal lavage cytology but negative macroscopic peritoneal implant? World J Surg. 2005;29:1131-4.

9. Japanese gastric cancer treatment guidelines 2010 (ver. 3). Gastr Cancer. 2011;14:113-23.

10. PATE Board. Gastric cancer treatment $\left(\mathrm{PDQ}{ }^{\circledR}\right)$ health rofessional version. National Cancer Institute: Bethesda; 2018.

11. Okabe H, Ueda S, Obama K, Hosogi H, Sakai Y. Induction chemotherapy with S-1 plus cisplatin followed by surgery for treatment of gastric cancer with peritoneal dissemination. Ann Surg Oncol. 2009;16:3227-36.

12. Kodera Y, Ito S, Mochizuki Y, Ohashi N, Tanaka C, Kobayashi D, et al. Long-term follow up of patients who were positive for peritoneal lavage cytology: final report from the CCOG0301 study. Gastr Cancer. 2012;15:335-7.

13. Aizawa M, Nashimoto A, Yabusaki H, Nakagawa S, Matsuki A, Homma K, et al. The clinical significance of potentially curative resection for gastric cancer following the clearance of free cancer cells in the peritoneal cavity by induction chemotherapy. Surg Today. 2015;45:611-7.

14. Yoshida K, Yamaguchi K, Okumura N, Tanahashi T, Kodera Y. Is conversion therapy possible in stage IV gastric cancer: the proposal of new biological categories of classification. Gastr Cancer. 2016;19:329-38.

15. Terashima M. Conversion therapy for gastric cancer: who can make conversion as successful as Goromaru? Gastr Cancer. 2016;19:685-6.

16. Leake PA, Cardoso R, Seevaratnam R, Lourenco L, Helyer L, Mahar A, et al. A systematic review of the accuracy and indications for diagnostic laparoscopy prior to curative-intent resection of gastric cancer. Gastr Cancer. 2012;15(Suppl 1):S38-47.

17. Tsuburaya A, Mizusawa J, Tanaka Y, Fukushima N, Nashimoto A, Sasako M. Neoadjuvant chemotherapy with S-1 and cisplatin followed by D2 gastrectomy with para-aortic lymph node dissection for gastric cancer with extensive lymph node metastasis. $\mathrm{Br}$ J Surg. 2014;101:653-60.

18. Kodera Y, Ito S, Mochizuki Y, Kondo K, Koshikawa K, Suzuki $\mathrm{N}$, et al. A phase II study of radical surgery followed by postoperative chemotherapy with S-1 for gastric carcinoma with free cancer cells in the peritoneal cavity (CCOG0301 study). Eur J Surg Oncol. 2009;35:1158-63.

19. Irino T, Sano T, Hiki N, Ohashi M, Nunobe S, Kumagai K, et al. Diagnostic staging laparoscopy in gastric cancer: a prospective cohort at a cancer institute in Japan. Surg Endosc. 2018:32:268-75.

20. Blank S, Lordick F, Bader F, Burian M, Dobritz M, Grenacher $\mathrm{L}$, et al. Post-therapeutic response evaluation by a combination of endoscopy and CT scan in esophagogastric adenocarcinoma after chemotherapy: better than its reputation. Gastr Cancer. 2015;18:314-25.

21. Eisenhauer EA, Therasse P, Bogaerts J, Schwartz LH, Sargent D, Ford R, et al. New response evaluation criteria in solid tumours: revised RECIST guideline (version 1.1). Eur J Cancer. 2009;45:228-47.

22. Nakamura M, Ojima T, Nakamori M, Katsuda M, Tsuji T, Hayata $\mathrm{K}$, et al. Conversion surgery for gastric cancer with peritoneal metastasis based on the diagnosis of second-look staging laparoscopy. J Gastrointest Surg. 2018. https://doi.org/10.1007/s1160 5-018-3983-7.

Publisher's Note Springer Nature remains neutral with regard to jurisdictional claims in published maps and institutional affiliations. 